\title{
Research on Mutation Parameters of Cloud Evolutionary Strategy
}

\author{
Ziqiang Luo ${ }^{1, a}$, Peng Cao ${ }^{2, b^{*}}$, Bin Wen ${ }^{1, c}$ and Yu Zhang ${ }^{1, d}$ \\ ${ }^{1}$ College of Information Science and Technology, Hainan Normal University, Haikou, 571158, China \\ ${ }^{2}$ Dept. of Mathematics and Physics, Qiongtai Teachers College, Haikou, 571100, China \\ a306003057@qq.com, blanyuan97@126.com, '951714238@qq.com, d344248003@qq.com
}

\section{${ }^{*}$ Corresponding Author}

Keywords: cloud model; heavy-tailed distribution; evolutionary strategy; mutation parameter.

Abstract. The cloud evolutionary strategy is a novel rapid evolutionary algorithm based on the outstanding characteristics of the cloud model on the process of transforming a qualitative concept to a set of quantitative numerical values. The paper conducts the mathematical analysis of cloud distribution with the change of mutation parameters, and studies some measures of dispersion of cloud distribution. This will be conducive to the further development and wide application of cloud evolutionary strategy.

\section{Introduction}

Cloud model is a new cognitive model for uncertain transformation between linguistic concepts and quantitative values $[1,2]$. We employ the expectation $E x$, the entropy En, and the hyper-entropy $H e$ to represent the concept as a whole. In the discourse universe, $E x$ is the position corresponding to the center of the cloud gravity, whose elements are fully compatible with the linguistic concept; En is a measure of the concept coverage, i.e. a measure of the fuzziness, which indicates how many elements could be accepted to the qualitative linguistic concept; and $\mathrm{He}$ is a measure of the dispersion on the cloud droplets, which can also be considered as the entropy of En.

At present, cloud model as an effective tool of uncertainty knowledge representation has been successfully applied to many areas, such as intelligent control, data mining, digital watermarking, subjective trust, credibility evaluation and evolutionary computation [3-8].

In the evolutionary strategy, the mutation operator is the major evolution operator. Gauss mutation, Cauchy mutation and uniform mutation are commonly used in real-coded representation. Ex can be defined as seed individual, which expresses the excellent characteristics of ancestral inheritance; $E n$ is defined as variation entropy, which approximately represents the evolution scope; and $\mathrm{He}$ is defined as variation hyper entropy, which reflects the stability of evolution. Obviously the more He/En, the greater the uncertainty. Though the change of mutation parameters ( $E n$ and $\mathrm{He}$ ), we can flexibly implement the transformation from Gauss mutation to uniform mutation in the cloud evolution strategy.

The paper is constructed as follows: Section II conducts the mathematical analysis of cloud distribution with the change of mutation parameters. Section III studies some measures of dispersion of cloud distribution.

\section{Mathematical analysis of cloud distribution}

In forward normal cloud, let domain $U=R, E n^{\prime} \sim N\left(E n, H e^{2}\right)(E n>0, H e>0)$, then the probability density function of $E n^{\prime}$ is

$$
f_{E n^{\prime}}(y)=\frac{1}{\sqrt{2 \pi} H e} e^{-\frac{(y-E n)^{2}}{2 H e^{2}}} .
$$

When $E n^{\prime}$ is constant value $y(\neq 0)$, the conditional probability density function of $X$ is 


$$
f_{X \mid E i}(x \mid y)=\frac{1}{\sqrt{2 \pi}|y|} e^{\frac{(x-E x)^{2}}{2 y^{2}}} \text {. }
$$

According to (1) and (2), and conditional probability density formula, the marginal probability density function of $X$ is

$$
f_{X}(x)=\int_{-\infty}^{+\infty} f_{X, E n^{\prime}}(x, y) d y=\int_{-\infty}^{+\infty} f_{E n^{\prime}}(y) \cdot f_{X \mid E n^{\prime}}(x \mid y) d y=\int_{-\infty}^{+\infty} \frac{1}{2 \pi H e|y|} e^{-\frac{(x-E x)^{2}}{2 y^{2}}-\frac{(y-E n)^{2}}{2 H e^{2}}} d y .
$$

Although we can get the analytical form of the marginal probability density function of $X$, the numerical solution of $f_{X}(x)$ and the cumulative probability density function $F_{X}(x)$ are easily computed by the method of kernel smoothing density estimate. The ksdensity in Matlab calls the function with a single argument that is an array containing distances between data values in $\mathrm{X}$ and locations in XI where the density is evaluated.

To facilitate statistical simulation, might as well assume $E x=0, E n=1, H e=0.1,0.2, \ldots, 60$, and the cloud droplets number $N=1000$, then we can get the numerical solution of $f_{X}(x)$ and $F_{X}(x)$, as shown in Fig. 1-3, where $\lambda=H e / E n$.

The figures indicate that the "peakedness" of the probability density function of $X$ increases sharply and declines rapidly again, then slowly decreases with the increase of $\lambda$. But the "tailedness" increases rapidly. It can be seen that with the increase of $\lambda$, we can flexibly implement the transformation from Gauss mutation to uniform mutation in the cloud evolution strategy.
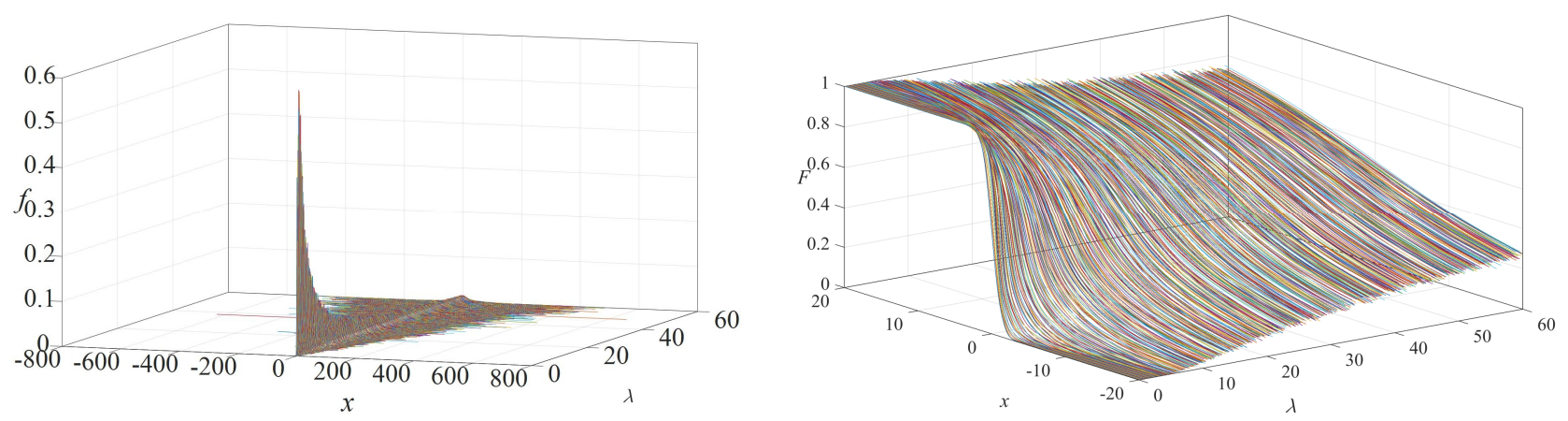

Fig. 1 the probability density function of $X$

Fig. 2 the cumulative probability density function of $X$

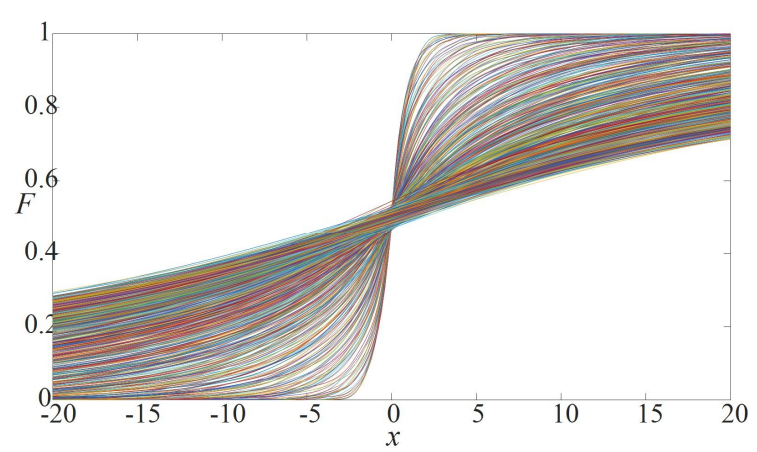

Fig. 3 the cumulative probability density function of $X$ at two-dimensional coordinates

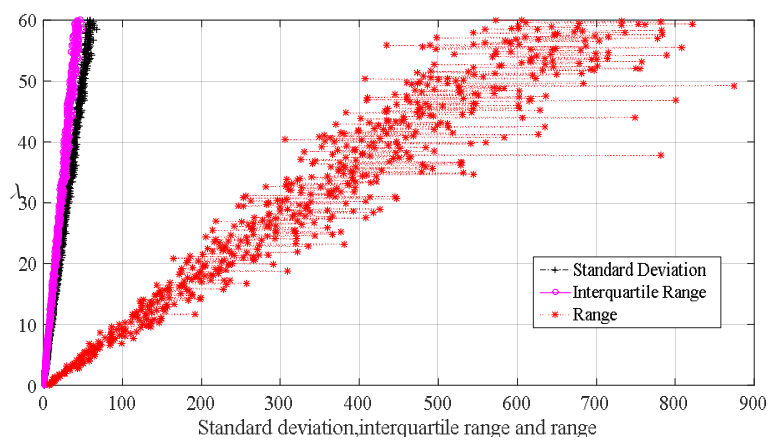

Fig. 4 the standard deviation, interquartile range and range of $X$

\section{Measures of dispersion of cloud distribution}

In statistics, dispersion (also called variability, scatter, or spread) denotes how stretched or squeezed a distribution (theoretical or that underlying a statistical sample) is. Some measures of statistical dispersion are conducted in-depth analysis and study by statistic simulation, such as the standard deviation, interquartile range and range kurtosis, as shown in Fig. 4. 
Standard deviation is the most commonly used measure of spread of data about the mean. The interquartile range is a method of measuring the spread of the numbers by finding the middle $50 \%$ of the values with the function of prctile().Interquartile range is defined as the difference between the 25 th and 75th percentile (also called the first and third quartile). If the interquartile range is large it means that the middle $50 \%$ of observations are spaced wide apart. It is the important advantage that interquartile range can be used as a measure of variability if the extreme values are not being recorded exactly. The range is the difference between the largest and the smallest observation in the data. The prime advantage of this measure of dispersion is that it is easy to calculate. But it is very sensitive to outliers and does not use all the observations in a data set. It is more informative to provide the minimum and the maximum values rather than providing the range. The fig. 4 indicates that with the increase of $\lambda$, the changes of standard deviation and interquartile range are far lower than the changes of range. It is thus clear that cloud mutation maintains the excellent characteristics of ancestral inheritance, and also can achieve better global optimization.

Kurtosis is a measure of the "tailedness" of the probability distribution of a real-valued random variable. The standard measure of kurtosis is based on a scaled version of the fourth moment of the data or population. This number measures heavy tails, and not peakedness; hence, the "peakedness" definition is misleading. For this measure, higher kurtosis means more of the variance is the result of infrequent extreme deviations, as opposed to frequent modestly sized deviations. The kurtosis of any univariate normal distribution is 3. It is common to compare the kurtosis of a distribution to this value. Distributions with kurtosis greater than 3 are said to be leptokurtic.

The kurtosis of $C(E x, E n, H e)$ is

$$
K(X)=E(X-E x)^{4} /(D X)^{2}=\frac{3 E n^{4}+18 E n^{2} H e^{2}+9 H e^{4}}{\left(E n^{2}+H e^{2}\right)^{2}}=\frac{3+18 \lambda^{2}+9 \lambda^{4}}{\left(1+\lambda^{2}\right)^{2}}=9-\frac{6}{\left(1+\lambda^{2}\right)^{2}} .
$$

It is obvious that $3<K(X)<9$, so normal cloud model is heavy-tailed distribution[9].It can be seen that with the increase of $\lambda$, the kurtosis of $X$ is quickly close to 9, as shown in Fig. 5 and Table 1.But with the increase of $\lambda$ from 0 to $9, X$ tends to conform to uniform distribution. Therefore the shape change of cloud distribution cannot be visually depicted by the kurtosis.

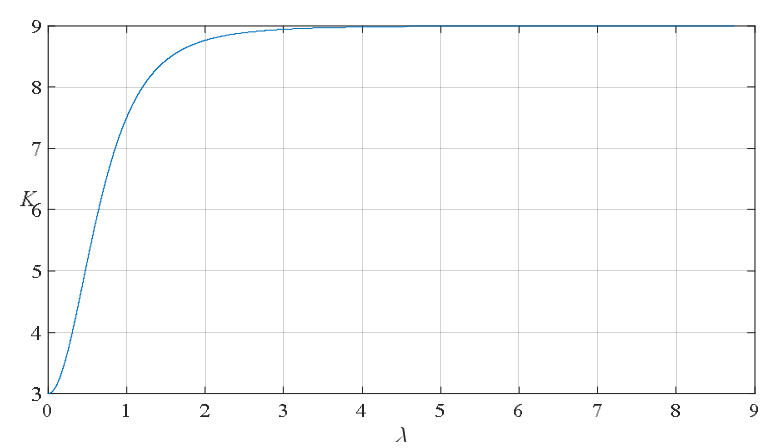

Fig. 5 the kurtosis of $X$ with changed $\lambda$

Table 1 the kurtosis of $X$ when $\lambda$ increases from 1 to 9

\begin{tabular}{|l|l|l|l|l|l|l|l|l|l|}
\hline$\lambda$ & 1 & 2 & 3 & 4 & 5 & 6 & 7 & 8 & 9 \\
\hline$K$ & 7.500 & 8.760 & 8.940 & 8.979 & 8.991 & 8.995 & 8.997 & 8.998 & 8.999 \\
& 0 & 0 & 0 & 2 & 1 & 6 & 6 & 5 & 1 \\
\hline
\end{tabular}

\section{Acknowledgment}

This work is supported by grants of National Nature and Science Foundation of China (No.61463012, No.61462025, No.61462024), Natural Science Foundation of Hainan Province (No.614233, 
No.114013, No.613161, No.613162), Qiongtai Teachers College Scientific Research Project (No.qtky201304).

\section{References}

[1] L. Deyi, D. Yi, Artificial Intelligence with Uncertainty, second ed., National Defense Industry Press, Beijing, 2014.

[2] L. Deyi, L. Changyu, G. Wenyan, A new cognitive model: cloud model, Int. J. Intell. Syst., 24(2009)357-375.

[3] Z. Guangwei, K. Jianchu L. Hesong, L. Deyi, Cloud model based algorithm for global optmization of functions, Journal of Beijing University of Aeronautics and Astronautics,33(2007) 486-490.

[4] Z. Guangwei, H. Rui, L.Yu, L. Deyi, An evolutionary algorithm based on cloud model, Chinese Journal of Computers, 31(2008)1082-1091.

[5] L. Yu, L. Deyi, Z. Guangwei, C. Guisheng, Atomized feature in cloud based evolutionary algorithm, Acta Electronica Sinica, 37(2009)1651-1658.

[6] L. Yu, L. Deyi, Statistics on atomized feature of normal cloud model, Journal of Beijing University of Aeronautics and Astronautics, 36(2010)1320-1324.

[7] H. ZhenFeng, X.FanLun, Cloud model based evolution strategy driven by kurtosis, Pattern Recognition and Artificial Intelligence, 25(2012) 205-212.

[8] L. Ziqiang, C. Peng, Research of the software system reliability allocation based on cloud evolutionary strategy, Ship Electronic Engineering , 32(2012) 102-104.

[9] L. Deyi, L. Changyu, G. Wenyan, Proof of the heavy-tailed property of normal cloud model, Engineering Sciences, 13(2011)20-23. 